\title{
Neuraxial Hematoma after Epidural Anesthesia. Is It Possible to Prevent or Detect It? Report of Two Cases
}

\author{
Rodrigo de Lima e Souza, TSA 1, Luiz Otávio Fernandes Andrade 22, Joaquim Belchior Silva, TSA ${ }^{3}$, \\ Luiz Antônio Carneiro da Silva, TSA 4
}

Summary: Souza RL, Andrade LOF, Silva JB, Silva LAC - Neuraxial Hematoma after Epidural Anesthesia. Is It Possible to Prevent or Detect It? Report of Two Cases.

Background and objectives: Spinal hematomas are rare and they affect the central nervous system. They can cause permanent neurologic sequelae and death if they are not treated properly. Early diagnosis and treatment are fundamental for a good neurologic prognostic. The objective of this report was to emphasize for anesthesiologists the importance of early diagnosis and treatment of spinal hematomas, besides improving their prevention.

Case reports: Case 1: The patient underwent epidural lumbar anesthesia for femoropopliteal revascularization. He was being treated with acetylsalicylic acid, clopidogrel, and enoxaparin, which were discontinued before the surgery. The patient developed paraplegia in the immediate postoperative period. Neurosurgical decompression was performed after the diagnosis, but without recovery in the long run. Case 2: The patient underwent epidural lumbar anesthesia for right knee osteotomy, without intercurrences. The patient remained without neurological complaints until approximately 48 hours after the surgery when he developed urinary retention, pain in the right lower limb, paresthesias, and difficulty moving both feet. The MRI showed an epidural lumbar hematoma, and the patient underwent immediate surgical decompression. He showed complete neurological recovery after 10 months of rehabilitation.

Conclusions: The clinical cases presented here showed different outcomes, indicating the importance of early diagnosis and treatment for a good evolution. Diagnosis by MRI with early decompression shortly after the development of the first clinical manifestations remains the standard treatment. Identification of patients at risk for neuraxial bleeding and change in anesthetic technique, as well as the establishment of postoperative neurologic evaluation protocols in patients undergoing neuraxial anesthesia can contribute for prevention of severe neurologic sequelae.

Keywords: Hematoma, epidural, spinal; Anesthesia, Epidural; Postoperative complications.

[Rev Bras Anestesiol 2011;61(2): 218-224] @Elsevier Editora Ltda.

\section{INTRODUCTION}

Spinal hematomas affect the central nervous system and although rare they can cause permanent neurologic sequelae, and even death, if they are not properly treated.

According to some authors, the incidence of spinal hematomas secondary to epidural anesthesia is small $(1: 168,000$ to $1: 190,000)$. However, it can vary according to the population. For example, the incidence of spinal hematoma in the elderly undergoing large orthopedic surgeries can reach 1:3,600 1,2.

According to some studies the relative risk of permanent neurologic damage after epidural anesthesia can vary from zero to $7.6: 10,000$ anesthesias ${ }^{3,4}$. In the obstetric population, the incidence of permanent or severe sequelae after epidural anesthesia is close to $1: 237,000^{1,2}$.

Received from Hospital Madre Teresa, Belo Horizonte, Minas Gerais, Brazil.

1. Anesthesiologist; Coordinator of Anesthesia Specialization of Hospital Madre Teresa; Surgery Professor

2. Anesthesiologist of Hospital Madre Teresa; Director of Supplies of UNIMED, Belo Horizonte

3. Anesthesiologist of Hospital Luxemburgo and of IPSEMG

4. Anesthesiologist of Hospital Madre Teresa and Hospital das Clínicas

Submitted on February 3, 2010

Approved on September 3, 2010.

Correspondence to:

Dr. Rodrigo de Lima e Souza

Rua Lauro Ferreira 163, apto 1.900

Buritis

30575-080 - Belo Horizonte, MG, Brazil

E-mail: digo7@terra.com.br
Regarding the etiology of spinal hematomas, in approximately one third of the cases the cause of bleeding is not identified, being considered idiopathic. In the remaining two thirds, prevention of identifiable causes is associated with some risk factors that can contribute for the development of spinal hematomas. Thus, identification of those risk factors for neuraxial bleeding is important in pre-anesthetic evaluation ${ }^{5}$. The most common risk factors include:

- Use of antihemostatic drugs (coumarins, anti-platelets, high- and low-molecular weight heparin, fibrinolytics).

- Presence of spinal vascular malformations.

- Family history of bleeding disorders or coagulopathies.

- History of abnormal bleeding in small surgeries (dental treatment, for example).

- Gingival, nasal, or skin bleeding without apparent cause.

- Severe liver and kidney disease.

- Disease of the spine (stenosis of the medullary canal, ankylosing spondylitis).

- Preeclampsia with liver dysfunction and thrombocytopenia.

- Hypertension and alcoholism.

Primordial care for prevention of neuraxial bleeding includes atraumatic puncture technique and introduction or removal of epidural catheters obeying the correct interval of suspension of antihemostatic drugs ${ }^{5,9}$. 
Besides, neuraxial blocks constitute only the fourth cause of spinal hematoma, and it is important to remember that in approximately $30 \%$ of the cases patients may not have coagulopathies or use of anticoagulants 5 .

Early diagnosis and treatment (in the first 6 to 12 hours after the development of symptoms), besides a milder initial presentation, are key factors for a good evolution, translating in fewer sequelae and better survival. On the other hand, delayed diagnosis and initial presentation with severe neurologic deficit could mean worse evolution and possibility of death or permanent sequelae ${ }^{5,6}$.

The objective of this report was to call the attention of anesthesiologists for the importance of early diagnosis and treatment of spinal hematomas, besides improving their prevention.

\section{CASE REPORTS}

\section{Case 1}

This is 79 years old male patient with median height and weighing approximately $70 \mathrm{~kg}$, physical status ASA 3, with hypertension and peripheral vasculopathy. An angiographic study showed severe peripheral vascular insufficiency and mild coronariopathy. He was admitted with intermittent claudication and angina to medium efforts. He had a history of recent left femoropopliteal revascularization. The patient was being treated with acetylsalicylic acid, clopidogrel, simvastatin, and enalapril. The exam showed occlusion of the left and right superficial femoral arteries, the presence of a pervious left femoropopliteal graft, besides severe bilateral occlusive lesions in the tibiofibular trunks.

After adequate clinical orientation, anti-platelet drugs were discontinued for seven days and low molecular weight heparin was administered until the day before the surgery for prophylaxis of thromboembolic events. Prior coagulation studies showed prothrombin activity of $72 \%$, INR 1.24 , and ATTP $30 / 30$ sec. Platelet count was $248,000 \cdot \mathrm{mm}^{-3}$, hematocrit of $40 \%$, and hemoglobin $13.1 \mathrm{mg}^{\mathrm{dL}}{ }^{-1}$. The remaining exams were normal.

Right femoropopliteal revascularization was performed under epidural anesthesia without intercurrences, respecting the safety interval between the last dose of enoxaparin and the puncture (over 12 hours). After the procedure, the patient was transferred to the postanesthetic care unit (PACU) where he remained under observation for four hours, when he showed complete recovery of sensitivity and motor skills in the lower limbs.

Twenty-four hours after the surgery the patient developed paraplegia, paresthesias, and sensorial loss for tactile, pressure, and temperature in the lower limbs and abdomen below the $T_{10}$ dermatome. The neurosurgical team was called and the patient was sent for an emergency thoracolumbar MRI. The exam clearly showed a posterior neuraxial hematoma from $T_{6}$ to $L_{2}$.

The patient was subsequently submitted to a medullar decompression surgery. During the surgery, a dural arteriovenous fistula was observed at the $L_{1}$ level, close to the level of the epidural puncture, suggesting a possible source of bleeding.
At the end of the anesthetic-surgical procedure, the patient was transferred to the PACU without resolution of the neurologic deficit, remaining in the same conditions after one year of rehabilitation therapy.

\section{Case 2}

This is a 48 years old male patient weighing $82 \mathrm{~kg}$ with non insulin-dependent diabetes mellitus in regular use of an oral anti-diabetic drug (metformin), physical status ASA 2, who underwent a right knee osteotomy due to advanced gonarthrosis. Preoperative exams were within normal limits (CBC, coagulogram, glucose levels, and ions). Epidural anesthesia with a single puncture at the $L_{3}-L_{4}$ level using $1 \%$ ropivacaína $(20 \mathrm{~mL})$ and morphine $(2 \mathrm{mg})$ was the anesthetic technique used without intercurrences. The patient was discharged from the PACU after 6 hours without complaints, moving the lower limbs and with preserved superficial and deep sensitivity.

$\mathrm{He}$ remained without pain, urinary, or lower limb motor complaints until approximately 48 hours after the surgery when he developed urinary retention, sciatic pain in the lower right limb, paresthesias, and difficulty moving his feet. He was examined by the neurosurgical team who confirmed the diagnosis of Cauda Equina syndrome. An MRI of the spine showed an ovoid formation $(3.6 \mathrm{~cm} \times 1.6 \mathrm{~cm})$ in the posterior epidural topography at the $\mathrm{L}_{3}-\mathrm{L}_{4}$ level, leading to stenosis of the vertebral canal.

The patient was then submitted to decompressive laminectomy in $L_{3}-L_{4}$ under general anesthesia, which confirmed the presence of a neuraxial hematoma at the same level.

The patient remained with changes in sphincter control, neuropathic pain, and paresthesias in the corresponding dermatomes for three months, besides weakness in his legs and feet for five months. Since then he evolved with improvement of perineal sensitivity, sphincter control, and neuropathic pain. However, he remained with difficulty walking due to incomplete dorsiflexion of the toes, which resulted in complete neurological recovery after three more months.

\section{DISCUSSION}

The incidence of neuraxial hematomas in patients undergoing neuraxial anesthesia is variable. For example, in a retrospective multicenter study in Sweden, the incidence of those hematomas after orthopedic surgeries ranged from $1: 3,600$ to $1: 29,000^{1}$.

The development of lower limb motor blockade in patients submitted to continuous epidural infusion is a trustworthy sign of progressive spinal hematoma and indicates a poor prognosis ${ }^{5,7}$. Based on this, early diagnosis is mandatory.

The clinical cases reported here demonstrated different outcomes, showing the importance of early diagnosis and treatment for a good evolution. In the first case, the lack of identification of early signs and symptoms of neuraxial compression most likely contributed for the poor outcome. 
However, the early diagnosis of compressive neuraxial hematoma remains difficult in daily medical practice since clinical manifestations may develop after the observation period in the PACU. As an example, in both cases presented here, the patients were released from the PACU with normal sensorial and motor exams of the lower limbs and perineal area, even after 6 hours of observation. Thus, according to a metanalysis of 613 patients with neuraxial hematoma, patients who developed symptoms after 24-36 hours show a tendency for a poorer and incomplete recovery, which is the opposite of those who develop symptoms up to 6 hours after the procedure ${ }^{5}$.

In an attempt to favor early diagnosis, Meikle et al. ${ }^{7}$ investigated centers which had mechanisms to detect and manage anesthesia-related neuraxial hematomas, and they demonstrated that only $55 \%$ of pain and anesthesia services had a protocol to detect signs and symptoms of abnormal recovery from neuraxial anesthesia. Besides, only $57 \%$ of those departments had MRI available 24 hours a day. Approximately one third of neuraxial hematomas were not diagnosed and treated within the initial 24 hours. The authors suggested protocols for routine neurologic evaluation for clinics of pain and anesthesia services, especially after the use and removal of epidural catheters. Some of those recommendations are listed below:

- patients with continuous epidural infusion should be observed for the presence of motor blockade at least every 4 hours.

- Those observations should continue for 24 hours after removal of the epidural catheter.

- An adequately trained person should be responsible for investigating signs suggestive of neuraxial hematoma.

- If in the absence of a recent bolus of local anesthetic there is a decline in motor function, the person responsible should be informed immediately.

- If the motor blockade is attributable to a recent dose of the local anesthetic, the patient should be reevaluated in 2 hours.

- If the presence of a neuraxial hematoma is suspected, an MRI should be performed and neurosurgical evaluation should be requested as soon as possible.

In the first clinical case, the presence of spinal vascular malformation, indentified posteriorly, corroborates this finding as an important cause of neuraxial bleeding ${ }^{5,10-13}$. In this context, neuraxial anesthesia is considered a triggering factor. Other factors identified in patients with neuraxial bleeding include congenital and acquired coagulopathies. Among the former, antihemostatic therapy is the main cause. The association of antihemostatic drugs and traumatic puncture can be a trigger factor for neuraxial bleeding ${ }^{5}$.

As a consequence, the anesthesiologist should be attentive in the presence of those factors and respect the recommended interval between the puncture and the administration of antihemostatic drugs (coumarins, anti-platelet drugs, high- and low-molecular weight heparin) ${ }^{8,9}$. Note that, similar to the two cases presented here, patients with acquired coagulopathies can have normal coagulation tests, which does not rule out the risk of developing neuraxial hematomas $3,5,12,14$.

Other general conditions associated to neuraxial anesthesia can also contribute for the development of neuraxial hematomas, such as: polytrauma, primary and metastatic spinal medullary tumors, Paget's disease, systemic lupus erythematosus, ankylosing spondylitis, and hypertension associated with atherosclerosis, among others ${ }^{12-14}$.

On the other hand, approximately $40 \%$ of neuraxial hematomas do not have an identifiable trigger factor ${ }^{5}$. Some of those factors may be trivial such as evacuation, and sexual or coughing efforts, or spontaneous, usually associated with local (vascular fragility, trauma, tumors) and/or systemic (atherosclerosis) predisposing factors. This contributes for the difficulty of adequate selection of candidates for neuraxial anesthesia.

In the last two decades in the United States, 1,005 medical lawsuits were associated with neuraxial anesthesia according to the data of the America Society of Anesthesiologists (ASA). Among those, spinal hematomas were responsible for $43 \%$ of the total complications associated with neuraxial anesthesia, resulting in $89 \%$ of permanent neurologic damage. Approximately $56 \%$ of those lawsuits resulted in indemnifications with a mean value of approximately US $\$ 183,500$. Besides, approximately three fourths of documented spinal hematomas in those lawsuits were related to intrinsic (for example, preeclampsia) or iatrogenic (use of antihemostatic drugs) coagulopathies. The mean time for the diagnosis was two days, and it was significantly higher than the mean time (one day) for the development of initial signs and symptoms $(\mathrm{p}<0.05)^{15}$.

Diagnosis by MRI and early decompression shortly after the development of the first clinical manifestations (within 12 hours) remain the standard treatment $5,7,13$. But some cases of neuraxial bleeding can be treated conservatively 6,10 . The decision to treat someone conservatively should be based on the progression of neurologic signs and symptoms and evaluation by a neurosurgeon.

Alternatively, the presence of a neurologic deficit after the neuraxial anesthesia can be secondary to several other causes not related to the presence of hematomas. For example, direct needle trauma during puncture, factors related to local anesthetics (type, dose and concentration, duration of exposure), the use of epinephrine, or accidental injection of substances inappropriate for epidural or subarachnoid use. Injury due to malposition of the patient in the operating table can also be mentioned ${ }^{11,16}$.

Identification of patients at risk for neuraxial bleeding, change in anesthetic technique, as well as establishing postoperative neurologic evaluation protocols for patients undergoing neuraxial anesthesia can contribute for the prevention of severe and permanent neurologic sequelae. 